\title{
Cathepsin B-activatable cell-penetrating peptides for imaging cancer-related Cathepsin B
}

Konstantin Kuhne ${ }^{1}$, Lydia Behring ${ }^{1}$, Birgit Belter ${ }^{1}$, Robert Wodtke ${ }^{1}$, Lara von Krüchten², Max Keller ${ }^{2}$, Jörg Steinbach ${ }^{1}$, Jens Pietzsch ${ }^{1}$, Reik Löser ${ }^{1}$

${ }^{1}$ Helmholtz-ZentrumDresden-Rossendorf, Institute of Radiopharmaceutical Cancer Research, Germany

${ }^{2}$ Institute of Pharmacy, Faculty of Chemistry and Pharmacy, University of Regensburg, Germany

https://doi.org/10.17952/35EPS.2018.052

In our understanding of the many drivers of malignant progression and cancer metastasis, proteases are increasingly drawn into the spotlight as crucial modulators in cancer angiogenesis, invasion, and metastasis.[1] Elevated activities of multiple members of the cathepsin family have been shown to correlate with increased metastasis and high therapy resistance.[2, 3] In particular, high expression levels of extracellular Cathepsin B (CatB) indicate poor prognosis in various neoplastic diseases, which makes $\mathrm{CatB}$ an interesting target for activity-based molecular imaging in cancer diagnostics as well as in cancer treatment monitoring for personalised therapies. It is our aim to develop such an imaging probe for CatB by combination of a polyarginine-based, activatable cell-penetrating peptide (ACPP), as first described by R. Tsien and coworkers, and an optimised endopeptidase substrate for CatB in one molecule.[4] Substrate optimisation proofed to be challenging as two opposite factors needed to be balanced: stability against serum proteases to prevent premature cleavage of the activation sequence, while retaining efficient and specific endoproteolytic cleavability by CatB.

We have generated a CatB-endoprotease substrate by C-terminally elongating the CatB carboxydipeptidase substrate $\mathrm{AbzGIVR}{ }^{\star} \mathrm{AK}(\mathrm{Dnp}) \mathrm{OH}$ (Abz aminobenzoyl; Dnp dinitrophenyl; * denominates CatB cleavage site), described by Cotrin et al. in 2004, to the octapeptide AbzGIVR ${ }^{\star} \mathrm{AK}(\mathrm{Dnp}) \mathrm{GXCONH}_{2}$, which can be used as activation site in the final ACPP.[5] Introduction of any amino acid other than glycine at the P4' position resulted in hysteretic kinetics for the CatB-catalysed hydrolysis of the octapeptides, which might indicate the displacement of the occluding loop from the active site upon interaction with the substrate. Valine was identified as the most efficient amino acid in the P4' position (Fig. 1).

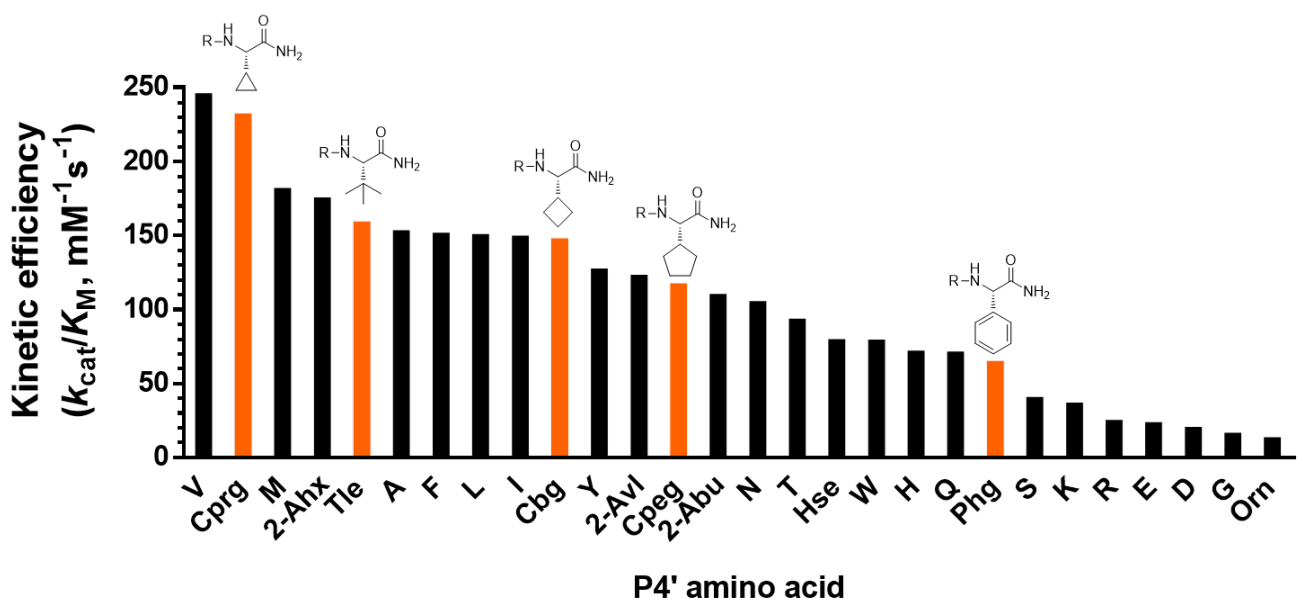

Figure 1: Effect of the P4' amino acid on the kinetic efficiency for the proteolytic cleavage by CatB. Experiments were performed using the model substrate Abz-GIVR*AK(Dnp)GX-CONH$H_{2}$, with Abz-Dnp forming a FRETpair and $X$ indicating the variability of the P4' position. Abbreviations: 2-Ahx 2aminohexanoic acid; 2 Avl 2-aminovaleric acid; Cbg cyclobutylglycine; Cpeg cyclopentylglycine; Cprg cyclopropylglycine; FRET fluorescence-resonance energy transfer; Hse homoserine; Orn ornithine; Phg phenylglycine; Tle tert-leucine.

Using LC-ESI-MS-based analysis of substrates incubated in human serum, the positions P1 and P3' where determined to be primary determinants of serum stability. After suppression of the unspecific cleavage at the P3' by $\mathrm{N}^{\alpha}$-methylation and optimisation within the positions P1-P3 (Fig. 2A), we were able to increase serum half-life from $<5$ min to $>24 \mathrm{~h}$ under concomitantimprovement of kinetic substrate efficiency towards CatB for substrates carrying the GFLG sequence at P4-P1 (Fig. 2B, Compounds3-5).[6]

Based on these results, we have synthesised an ACPP mirroring Tsien's original design: A nonaDarginine CPP, 
carrying 6-carboxytetramethylrhodamine (6TAM) as reporter group, is linked via our optimised CatB-substrate (as activation sequence) to a nonaDglutamate attenuation sequence (Fig. 3A). Cellular uptake was evaluated using CatB-expressing humanglioblastoma cells (U87MG).[7] After activation, the ACPP-6TAM accumulated rapidly within the nucleolus, independent of experimental conditions (Fig. 3B). This was in good accordance with literature, as Martin et al. described nucleolus-specific accumulation for polyarginineCPPs containing at least six arginines.[8]

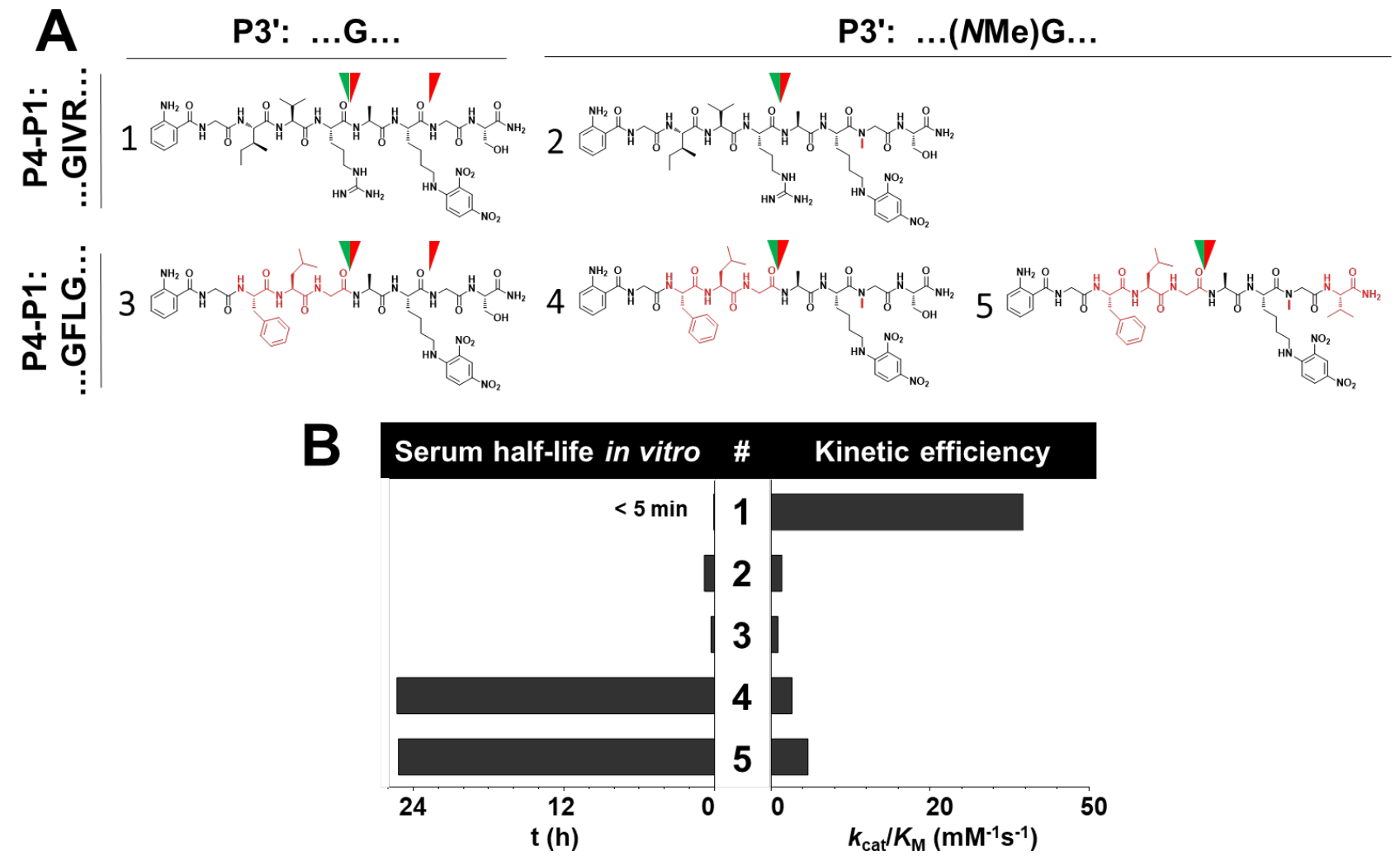

Figure 2: CatB-substrates evaluated for optimised stability by in vitro serum stability assay using human serum. Green half-arrow heads indicate CatB cleavage site, red half-arrow heads indicate unspecific cleavage site from human serum proteases identified by LC-ESI-MS fragment a nalysis. (B) Combination plot of serum half-life in vitro versus kinetic efficiency for substrate cleavage by CatB. Identifiers correspond to substrate structure in (A).

ACPP-6TAM activation and subsequent uptake was found to be dependent on both temperature(Fig. 3C, blue vs. red boxes) and the presence of $0.5 \mu \mathrm{M}$ dithiothreitol(DTT, Fig. 3C, filled vs. unfilled red boxes), indicating a biocatalytic process which is additionally dependent on a reductive environment - as is necessary for continuous CatB-activity during in vitro assays. [5] Performing the cellular uptake experiment in the presence of either broadrange Cys-protease and Cys-cathepsin inhibitors (Leupeptin and E64[9]) as well as the CatB-specific inhibitor CA074[10] resulted in significantly reduced uptake, while the a ddition of different inhibitorsf or cathepsin S (Gue2313[11]), cathepsin K (Odanacatib[12]), Asp-proteases (Pepstatin A) or matrix metalloproteases (Ilomastat[13]) had no effect on the uptake of the ACPP-6TAM. We therefore assume that the activation and subsequent uptake of the ACPP-6TAM is a specific, CatB-dependent process. 

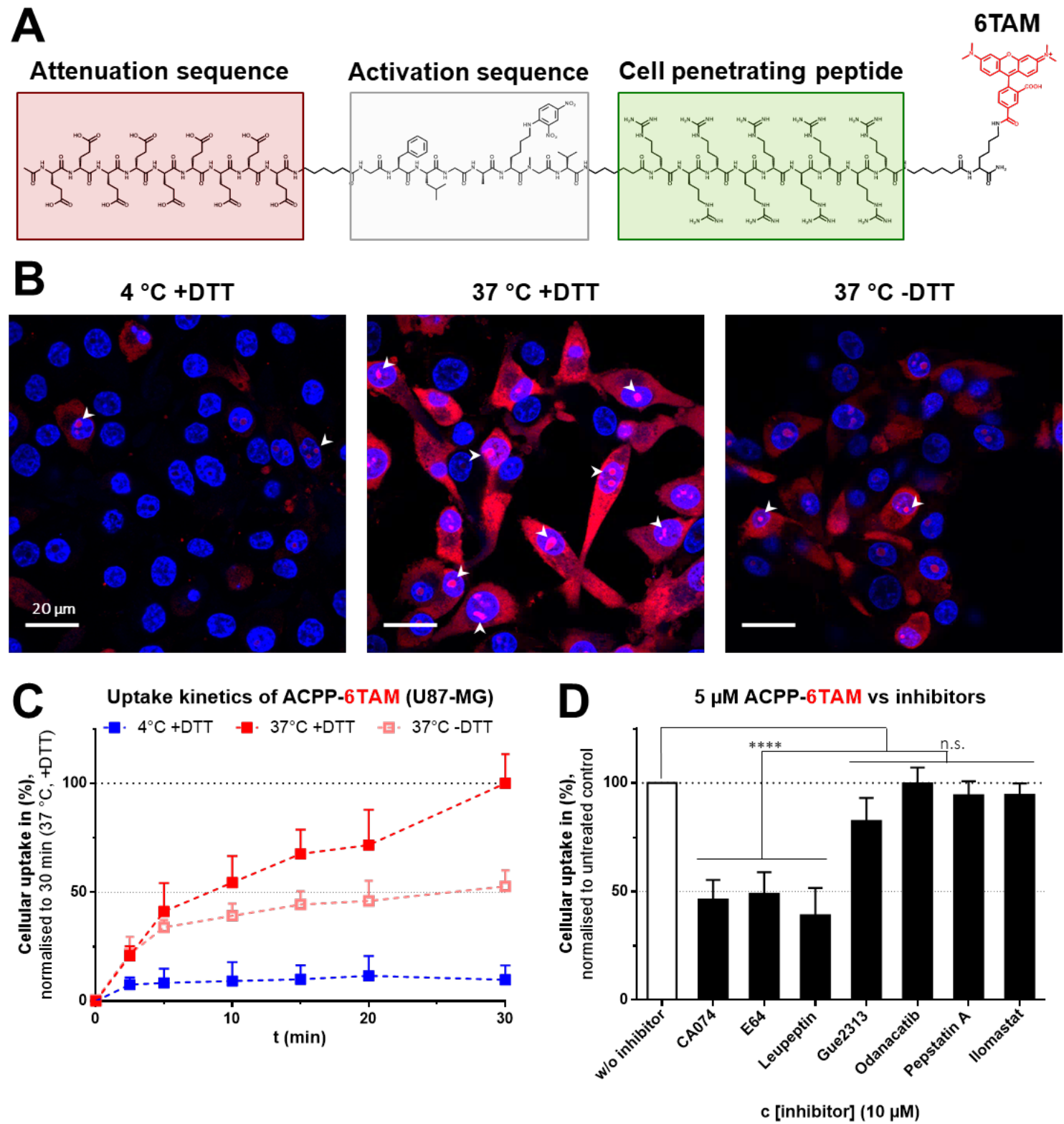

Figure 3: (A) Structure of the activatable cell-penetrating peptide. The N-terminal nona-d-glutamate attenuation sequence is highlighted by a red box, the central activation sequence (CatB substrate sequence) by a grey box and the cellpenetrating nona-d-arginine sequence by a green box, with 6TAM (red) linked to a $C$-terminal lysine residue. 6-Aminohexanoic acid spacers were used to confer flexibility between s u bunits. (B) Cellular uptake of ACPP-6TAM in U87MG cells. Intra-nucleolar accumulation of CPP(6TAM)-fragment indicated by white arrowheads. Nuclear stain: Hoechst 33342. (C) Kinetics of the cellular uptake of $5 \mu M$ ACPP-6TAM conjugate in U87MG cells. Uptake is strongly dependent on temperature $\left(4^{\circ} \mathrm{C},+D T T\right.$ vs. $\left.37^{\circ} \mathrm{C},+D T T\right)$ and on the addition of $0.5 \mathrm{mM} \mathrm{DTT}\left(37^{\circ} \mathrm{C},+D T T\right.$ vs. $\left.37^{\circ} \mathrm{C},-\mathrm{DTT}\right)$, both indicating a biocatalytic process responsible for the ACPP activation and uptake. Mean 6TAM-fluorescence values were normalised to nuclear stain for each image. Corresponding ratios were then normalised to untreated cells $(=0 \%)$ and treatment 37 ${ }^{\circ} \mathrm{C},+D T T(=100 \%)$ for each experiment. $n=3, N \geq 6$. (D) CatB-selective inhibition of the cellular uptake of ACPP-6TAM in U87MG cells. Cellular uptake $\left(15 \mathrm{~min}, 37^{\circ} \mathrm{C},+D T T\right)$ of $5 \mu M$ ACPP-6TAM in U87MG cells was significantly reduced by the addition of CA074 (CatB-selective inhibitor), E64 (broad-range cathepsin inhibitor) as well as Leupeptin (broad-range Cys-, Ser- and Thr-protease inhibitor). In contrast, the addition of either Gue2313 (cathepsin S-selective inhibitor), Odanacatib (cathepsin K-selective inhibitor), Pepstatin A (Asp-protease inhibitor) or Ilomastat (broad-range MMP inhibitor) did not result in a significant reduction of cellular uptake. Inhibitors were used at $c=10 \mu \mathrm{M}$. Data analysis performed as described in previous section. $n$ $=3, N \geq 6$. 


\section{References}

[1] Yang et al. Cancer Growth Metastasis 2009, 2, 13-27;

[2] Aggarwal and Sloane Proteomics Clin. Appl. 2014, 8, 427-437;

[3] Löser and Pietzsch Front. Chem. 2015, 3, article 37;

[4] Jiang et al. Proc. Natl. Acad. Sci. USA 2004, 101, 17867-17872;

[5] Cotrin et al. Anal. Biochem. 2004, 335, 244-252;

[6] Duncan et al. Br. J. Cancer 1987, 55, 165-174

[7] Yan et al. DNA Cell Biol. 2000, 19, 79-91;

[8] Martin et al. Nucleus 2015, 6, 314-325;

[9] Hanada et al. Agric. Biol. Chem. 1978 42, 523-528;

[10] Murata et al. FEBS Lett. 1991, 280, 307-310;

[11] Frizler et al. J. Med. Chem. 2012, 55, 5982-5986;

[12] Gauthier et al. Bioorg. Med. Chem. Lett. 2008, 18, 923-928;

[13] Grobelny et al. Biochemistry 1992, 31, 7152-7154 